Paediatr. Paedolog. 2019 · 54:74-80 https://doi.org/10.1007/s00608-019-0655-0 Online publiziert: 26. Februar 2019 (c) Der/die Autor(en) 2019

CrossMark

\author{
Martin Fuchs ${ }^{1,2}$ \\ ' Abteilung für Kinder- und Jugendpsychiatrie, Psychotherapie und Psychosomatik, Tirol Kliniken, Hall i. T., \\ Österreich \\ ${ }^{2}$ Medical University of Innsbruck, Innsbruck, Österreich
}

\title{
Cannabis, Badesalze und das Internet
}

\section{Alte und neue Drogen bei Kindern und Jugendlichen}

\section{Prävalenz von jugendlichem Drogengebrauch}

Eine Einschätzung der Prävalenz von jugendlichem Drogengebrauch ist methodisch schwierig. Aufgrund der thematisch inhärenten gesellschaftlichen Kontrollmechanismen (illegales Verhalten wird abgefragt) ist in entsprechenden Erhebungen mit einem erheblichen Dunkelfeld und einer damit verbundenen Unterschätzung zu rechnen [1]. Dennoch erlauben groß angelegte $\mathrm{Be}$ fragungen, wie z.B. die Erhebung des European School Survey Project on Alcohol and other Drugs (ESPAD), eine Einschätzung von Häufigkeit und zeitlicher Entwicklung von Drogenkonsum im Jugendalter. Die ESPAD-Erhebung wurde zwischen 1995 und 2015 insgesamt 6-mal in 48 europäischen Ländern durchgeführt; 2003, 2007 und 2015 wurden auch österreichische Jugendliche mit eingeschlossen. Dadurch ist es möglich, das Konsumverhalten österreichischer junger Menschen sowohl im longitudinalen Verlauf zu beurteilen, als auch in einen Vergleich mit anderen europäischen Ländern $\mathrm{zu}$ setzen. In die aktuelle Erhebung wurden insgesamt 96.046 europäische Jugendliche des Geburtsjahrgangs 1999 eingeschlossen, davon 8044 aus ganz Österreich. Aus methodischen Gründen (leichte Erreichbarkeit) wurden in die ESPADStudien nur Schüler eingeschlossen, was

Nach einem Vortrag im Rahmen der 55. ÖGKJ Tagung, Graz 2017. zusammen mit dem bereits erwähnten Dunkelfeldeffekt ebenfalls zu einer möglichen Unterschätzung der Konsumerfahrungen führen könnte: Problematischer Substanzkonsum ist häufig mit psychosozialen Problemen wie z. B. einem Abbruch des Ausbildungsverhältnisses verbunden; somit werden konsumierende Jugendliche von einer Schulstudie u. U. nicht gut erfasst [2].

In $\bullet$ Tab. 1 werden die 2015 erhobenen Prävalenzzahlen sowie der jeweilige europäische Durchschnittswert dargestellt.

Angesichts dieser Daten kann festgehalten werden, dass die von österreichischen Jugendlichen angegebenen Konsumerfahrungen in den meisten Bereichen über dem europäischen Durchschnitt liegen, bezüglich des Konsums von Zigaretten und Alkohol lagen österreichische Jugendliche 2015 sogar im europäischen Spitzenfeld. Erfreulichere Resultate zeigen longitudinale Analysen der 3 ESPAD-Erhebungszeitpunkte 2003, 2007 und 2015: Hier zeigt sich ein eindeutiger Rückgang des Alkohol- und Nikotinkonsums seit 2003 bei österreichischen Jugendlichen, sowohl was die Lebenszeitprävalenz (LTP) als auch was die Monatsprävalenz (LMP) betrifft. Dieser an sich begrüßenswerte Trend ist aber derzeit offenbar noch nicht stark genug, um österreichische Jugendliche aus dem europäischen Spitzenfeld hinsichtlich Alkohol und Nikotin zu verdrängen. Ein weitgehend stabiles Konsumverhalten zeigt sich seit über einem Jahrzehnt für Cannabis: Hier liegt die Lebenszeiterfah- rung von österreichischen Jugendlichen seit 2003 bei relativ konstanten $20 \%$ [2].

\section{Trends in Österreich}

Der jährlich erscheinende „Bericht zur Drogensituation" der Gesundheit Österreich $\mathrm{GmbH}$ stellt für das Berichtsjahr 2018 mehrere sich weiterhin abzeichnende Trends fest [3]. Zum einen ist weiterhin ein deutlicher Rückgang des risikoreichen Opioidkonsums bei unter 25-Jährigen zu verzeichnen; diese Droge scheint bei Jugendlichen nur mehr eine sehr untergeordnete Rolle zu spielen. Ergänzend dazu seien Rückmeldungen an den Autor aus mehreren Bundesländern inklusive Wien angeführt, die nahelegen, dass derzeit österreichweit nur mehr eine einstellige Zahl von Menschen unter 18 Jahren in oraler Opiatsubstitution befindlich ist. Zum zweiten scheint sich beim jugendlichen Probierkonsum das Substanzspektrum zu verbreitern, sodass Jugendliche heute bereit sind, mit einer Vielzahl unterschiedlicher Substanzen zu experimentieren. Zum dritten häufen sich in Europa und auch in Österreich Zwischenfälle in Zusammenhang mit unüblich hochdosiertem 3,4-Methylendioxy-N-methylamphetamin (MDMA) in Ecstasy-Tabletten [3, 4]. Die Einrichtungen checkit! in Wien (https:// checkit.wien) bzw. MDA-Basecamp in Tirol (http://www.drogenarbeitz6.at/ mda.html) bieten eine anonyme und straffreie Testung von Substanzen ab. Dabei wurden in abgegebenen EcstasyTabletten Dosierungen von bis zu $300 \mathrm{mg}$ 
Tab. 1 Konsumerfahrung, Erhebung der European School Survey Project on Alcohol and other Drugs (ESPAD) 2015 (Geburtskohorte 1999; Fragebogen)

\begin{tabular}{l|l|l}
\hline Substanz & $\begin{array}{l}\text { Prävalenz } \\
\text { Österreich (\%) }\end{array}$ & $\begin{array}{l}\text { Durchschnitt } \\
\text { Europa (\%) }\end{array}$ \\
\hline Zigarettenkonsum (LTP) & 53 & 46 \\
\hline Zigarettenkonsum (LMP) & 28 & 21 \\
\hline Alkohol (LTP) & 88 & 80 \\
\hline Alkohol (LMP) & 68 & 48 \\
\hline Intoxikation mit Alkohol (LMP) & 21 & 13 \\
\hline Illegale Drogen insgesamt (LTP): & 21 & 18 \\
\hline nur Mädchen & 19 & 15 \\
nur Buben & 23 & 21 \\
\hline Cannabis (LTP) & 20 & 16 \\
\hline MDMA (LTP) & 2 & 2 \\
\hline LTP Lebenszeitprävalenz; LMP Monatsprävalenz; & MDMA 3,4-Methylendioxy-N-methylamphetamin \\
\hline
\end{tabular}

pro Tablette gefunden. Entsprechende Safer-Use-Ratschläge gehen davon aus, dass eine Dosierung von 1,3 mg MDMA pro Kilogramm Körpergewicht bei Frauen und 1,5 mg MDMA pro Kilogramm Körpergewicht bei Männern nicht überschritten werden sollte. Somit liegen viele der derzeit abgegebenen Tabletten in einem toxischen Bereich.

\section{Cannabis}

Wie bereits erwähnt ist Cannabis sowohl bei Erwachsenen als auch bei Jugendlichen die mit Abstand am meisten verbreitete illegale Droge. Schätzungen gehen davon aus, dass in etwa die Hälfte der österreichischen Bevölkerung einmal in ihrem Leben Erfahrungen mit Cannabis machte bzw. machen wird. Im Jugendalter liegt die Lebenszeitprävalenz bei etwa $20 \%, 17 \%$ der befragten Jugendlichen gaben an, im letzten Jahr zumindest einmal Cannabis konsumiert zu haben, während etwa $10 \%$ angaben, auch im letzten Monat Cannabisprodukte konsumiert zu haben. Die Prävalenz liegt dabei für weibliche Jugendliche immer etwas niedriger als für männliche Jugendliche. Das durchschnittliche Einstiegsalter für Cannabis liegt deutlich höher als bei Alkohol und Nikotin rund um das 16. Lebensjahr, knapp $40 \%$ der befragten österreichischen Jugendlichen geben an, Cannabisprodukte seien sehr bzw. ziemlich leicht verfügbar [2].

Seit 2012 wird weltweit der nichtmedizinische Freizeitgebrauch von Cannabis legalisiert, auf 8 US-amerikanische Bun- desstaaten folgten 2014 Uruguay sowie 2018 Kanada mit entsprechenden Gesetzesbestimmungen für Erwachsene ab 18 bzw. 21 Jahren. Dieser Schritt wird in der Literatur kontrovers beurteilt, verschiedene Autoren kritisieren unter anderem die Entstehung einer mächtigen und primär auf Gewinn ausgerichteten Cannabis-Industrie. Diese könnte - in Analogie zur Tabak-Industrie - massiv an der Gewinnung neuer Kunden und damit wenig an der öffentlichen Gesundheit interessiert sein [5]. Wiederum andere Stimmen sprechen von einem Versagen des bisherigen internationalen Kurses der Drogenbekämpfung und fordern einen Paradigmenwechsel [5]. Die Idee, dass Prohibition eher zu Entstehung von hochpotenten Drogen führt, da sie leichter zu transportieren und zu verstecken sind, und Legalisierung diesem Effekt entgegenwirken könnte, scheint sich zumindest in den USA nicht gänzlich bewahrheitet zu haben. Im Gegenteil: der dortige Markt wird seit der gesetzlichen Öffnung von einer Vielzahl hochpotenter Cannabiskonzentrate überschwemmt, die von einer Industrie in Goldgräberstimmung zusammen mit anderen bisher nicht in der Form erhältlichen Produkten wie Cannabisschokolade, Cannabislimonade und sonstigen „edibles“ entwickelt werden. Es ist nicht auszuschließen, dass diese - für Erwachsene gedachten Produkte - auch abseits des vorgesehenen legalen Rahmens von Jugendlichen konsumiert werden [5]. Unabhängig von der US-amerikanischen Situation scheint sich insgesamt der Tetrahydrocannabinol(THC)-
Gehalt in Schwarzmarktprodukten (Cannabiskraut und Cannabisharz) in Europa im Lauf der letzten 25 Jahre deutlich erhöht zu haben [6].

Während gelegentlicher Freizeitkonsum von Cannabis bei Erwachsenen mit abgeschlossener Hirnreifung und ohne Risikoprofil für psychische Störungen relativ harmlos sein dürfte, ist die Datenlage bei Jugendlichen diesbezüglich gegenteilig. Zahlreiche Studien geben Hinweise auf eine Assoziation von jugendlichem Cannabiskonsum mit schädlichen psychischen Langzeitfolgen (kognitiv, affektiv) sowie mit psychischen Störungen wie Psychosen, Suizidalität, Suchterkrankungen sowie Angststörungen im Erwachsenenalter. Eine eindeutige Korrelation dieser möglichen schädlichen Langzeitfolgen besteht mit Einstiegsalter sowie Stärke des Konsums im Sinn einer drastischen Risikoerhöhung bei besonders jung einsetzendem Intensivkonsum. Einschränkend muss an dieser Stelle angemerkt werden, dass ein monokausaler Zusammenhang dieser Langzeitfolgen mit Cannabis als alleinigem Risikofaktor durch bisher vorliegende Studien nicht gezeigt werden kann. Zukünftige Forschung wird sich daher auch auf die Rolle genetischer Risikoprofile sowie komplexer Risikomodelle beziehen müssen. Tierexperimentelle Befunde geben allerdings klare Hinweise darauf, dass adoleszenter Cannabiskonsum irreversible schädliche Auswirkungen auf die Gehirnentwicklung haben kann und sich das diesbezüglich offenbar hochsensible jugendliche Gehirn vom erwachsenen und bereits entwickelten Gehirn unterscheidet. Das körpereigene Endocannabinoidsystem spielt eine wichtige Rolle für physiologische Reifungsmechanismen wie dem „pruning“ (Elimination unwichtiger Synapsen) oder dem Wachstum der weißen Gehirnsubstanz. Eine Beeinflussung dieser Prozesse durch jugendlichen Cannabiskonsum könnte also in dieser Schlüsselphase der Gehirnentwicklung besonders schädliche Auswirkungen hervorrufen $[7,8]$.

Von den zahlreichen Cannabinoiden, deren Wirkung schrittweise entschlüsselt wird, scheint vor allem das Zusammenspiel der zwei Inhaltsstoffe THC sowie Cannabidiol (CBD) von großer 
Bedeutung zu sein. Während THC berauschend, psychoaktiv, euphorisierend oder sedierend wirkt, aber auch Angst, Paranoia und psychosewertige Symptome erzeugen kann, wirkt CBD nicht psychoaktiv aber anxiolytisch und antipsychotisch und modifiziert die THCWirkung. Der Konsum industrieller Züchtungen mit extremem THC-Gehalt zuungunsten des CBD-Gehalts ist in diesem Zusammenhang sehr kritisch zu werten und wurde in einigen Studien mit einem erhöhten Psychoserisiko in Verbindung gebracht [9].

Immer wieder wird von Cannabisbefürwortern das therapeutische Potenzial dieser „ältesten Heilpflanze der Welt“ ins Treffen gebracht, und auf spektakuläre Einzelfälle verwiesen, wo die Verabreichung von entsprechenden Zubereitungen in bisher aussichtslosen Fällen eine Heilung bewirkt hätte. Die wissenschaftliche Datenlage hinsichtlich der medizinischen Nutzung von Cannabis im Kindes- und Jugendalter ist jedoch nach wie vor dürftig, kontrollierte Studien mit größerer Fallzahl und definierten pharmakologischen Eigenschaften der verabreichten Cannabinoide sind kaum verfügbar. Mögliche therapeutische Wirkungen, die durch das Endocannabinoidsystem vermittelt werden könnten, sind allerdings vielversprechend und reichen von antiemetischen, analgetischen, antiinflammatorischen, antiepileptischen, muskelrelaxierenden bis hin zu appetitsteigernden und stimmungsaufhellenden Effekten [10]. Das körpereigene Cannabinoidsystem ist kompliziert, zusätzlich zu den 2 bereits identifizierten Rezeptoren CB-1 und $\mathrm{CB}-2$ scheinen sich Endocannabinoide gegenseitig $\mathrm{zu}$ modifizieren und das bereits erwähnte CBD bindet beispielsweise nicht direkt an CB-1 oder CB-2, sondern scheint seine Wirkung über einen bisher unbekannten indirekten Mechanismus zu entfalten [11]. Somit können nur weitere Studien den Weg von positiven Einzelfallberichten hin zu einer kontrollierten und indikationsbezogenen Anwendung von Cannabinoiden im Kindes- und Jugendalter ebnen.

Paediatr. Paedolog. 2019·54:74-80 https://doi.org/10.1007/s00608-019-0655-0

(c) Der/die Autor(en) 2019

\section{Fuchs \\ Cannabis, Badesalze und das Internet. Alte und neue Drogen bei Kindern und Jugendlichen}

\begin{abstract}
Zusammenfassung
Die Art und Weise, wie österreichische junge Menschen aktuell legale und illegale Drogen konsumieren, sowie das Spektrum der verwendeten Substanzen hat sich in den letzten Jahren stark geändert. Neben der laufenden Cannabisdiskussion ist mit dem suchtartigen Gebrauch des Internets und damit assoziierter digitaler Medien ein neuartiges Gesundheitsproblem hinzugekommen. Substanzen wie 3,4-Methylendioxy$\mathrm{N}$-methylamphetamin („Ecstasy“) erfreuen sich ungebrochener Beliebtheit und sind in immer hochdosierterer und damit potenziell tödlicher Form verfügbar. Andere Substanzen wie Opiate spielen derzeit
\end{abstract}

bei österreichischen Jugendlichen in der Gesamtheit kaum eine Rolle. Dennoch kommt es immer wieder zu Todesfällen von jungen Menschen durch hochriskanten Mischkonsum unter Benzodiazepin- bzw. Opiatbeteiligung. Im folgenden Beitrag sollen im Überblick verfügbare Daten zu aktuellen Konsummustern österreichischer Jugendlicher dargestellt werden.

\section{Schlüsselwörter}

Drogen · Jugendliche · Internet · Cannabis . Prävention · Prävalenz · Österreich . Konsummuster

\section{Cannabis, Bath Salts and the Internet. Old and New Drugs in Children and Adolescents}

\section{Abstract}

The way in which young Austrians are currently consuming legal and illegal drugs and the spectrum of substances consumed have changed dramatically in the past few years. In addition to the ongoing cannabis debate, the addictive use of the internet and associated digital media has been recognized as a new health-related issue. Substances such as 3,4-methylenedioxymethamphetamine ("ecstasy") enjoy lasting popularity, and are available in rising and thus potentially deadly forms. Nowadays, other substances, such as opiates, are collectively barely an issue for
Austrian adolescents. However, the highrisk co-abuse among benzodiazepine/opiate users is still leading to fatalities among young people. The following article is aimed at giving an overview of the available data on the current consumption patterns in Austrian adolescents.

\section{Keywords}

Drugs · Adolescents · Internet · Cannabis · Prevention · Prevalence · Austria · Consumption patterns

\section{Badesalze}

Die Gruppe der sog. neuen psychoaktiven Substanzen (NPS), früher auch „research chemicals“ (RC) oder Legal Highs genannt, gelangte Anfang 2000 als sog. Badesalz-Drogen oder Bath Salts auf die Drogenmärkte in den USA und Europa. Aufgrund anfangs fehlender gesetzlicher Bestimmungen wurden die Substanzen zunächst teilweise legal vertrieben und z.B. als „Badesalze zur äußerlichen Anwendung" verkauft. Ab etwa 2010 wurden NPS in praktisch allen westlichen Ländern verboten. In Österreich wurde der Besitz, Konsum und Verkauf dieser Substanzen durch das Neue psychoaktive Substanzen Gesetz (NPSG) ab 2012 geregelt und unter Strafe gestellt.

NPS werden mittlerweile hauptsächlich über elektronische Marktplätze im Internet vertrieben. Diese sind meist an erschwert zugänglichen Stellen des Internets (sog. Darknet) lokalisiert. Für jugendliche Konsumenten sind diese $\mathrm{Zu}$ gangswege attraktiv, da unter Umgehung klassischer Straßen-Dealer ein Drogenkauf möglich ist. Bezahlt wird an diesen elektronischen Marktplätzen mithilfe von Kryptowährung (z. B. Bitcoins), die Drogen werden meist per Post in kleinen 
Päckchen nach Hause oder an ein Postfach zugestellt. In Österreich ist dieser Vertriebsweg auf dem Weg von einem Trend zu einem gängigen Kriminalitätsmuster [12]. NPS sind rein synthetisch hergestellte Substanzen und können grob in 5 Kategorien unterteilt werden [13]:

- Psychostimulanzien bzw. Amphetaminderivate (z.B. Mephedron oder Bath Salt),

- synthetische Cannabinoide (Spice, Räuchermischungen),

- benzodiazepinartige Substanzen,

- Dissoziativa (Ketaminanaloga) und

- Psychedelika (chemische Nachbauten von LSD, Psilobycin usw.).

Akute Effekte, Langzeitauswirkungen und empfohlener Einnahmemodus dieser Substanzen sind meist völlig unbekannt, jugendliche User gehen somit ein hohes Risiko ein und können sich nie im Klaren sein, was sie tatsächlich konsumieren [13]. Die Einrichtungen checkit! und $M D A$-Basecamp zeigen laufend, dass ein hoher Grad an Verunreinigungen vorliegt, außerdem häufig völlig andere Substanzen in den abgegebenen Proben getestet wurden, als die Konsumenten glaubten. Der Konsum von NPS scheint bei österreichischen Jugendlichen erfreulicherweise eine Randerscheinung zu sein [3].

\section{Internet - suchtartiger Gebrauch}

Das Internet spielt in der aktuellen Drogendiskussion bei jungen Menschen in 2 Bereichen eine wichtige Rolle. Einerseits dient es wie bereits dargestellt als Marktplatz, der sich speziell bei jugendlichen Konsumenten aber auch bei professionell agierenden kriminellen Organisationen immer größerer Beliebtheit erfreut [12]. Zum zweiten ist mit dem dysfunktionalen oder suchtartigen $\mathrm{Ge}$ brauch des Internets und damit assoziierter digitaler Medien ein neues Gesundheitsproblem entstanden.

Das Smartphone hat sich zu einem zentralen Alltagsgegenstand von jungen Menschen entwickelt: Innerhalb der letzten Jahre verdoppelte sich der Anteil der jugendlichen User von knapp 50\% im Jahr 2012 auf knapp 100 \% im Jahr 2016.
Hier steht eine Anzeige.

\section{Springer}




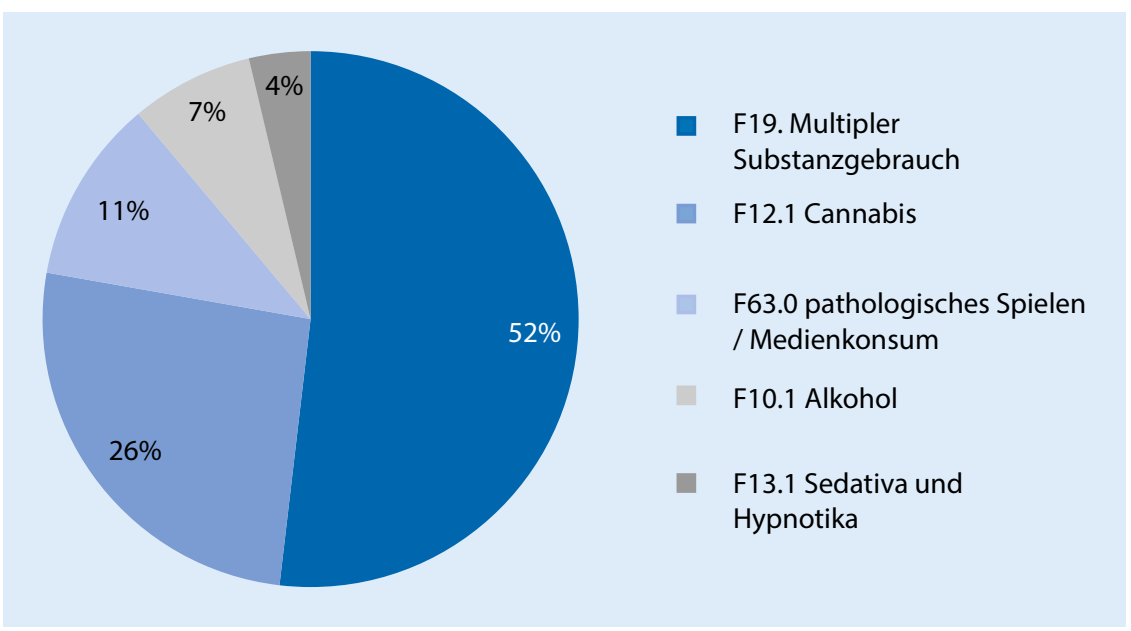

Abb. 1 ॥ Diagnoseverteilung bisheriger stationärer Patienten $(n=27)$. (Daten zur Verfügung gestellt von Mag. Natalie Youssefi [Hall])

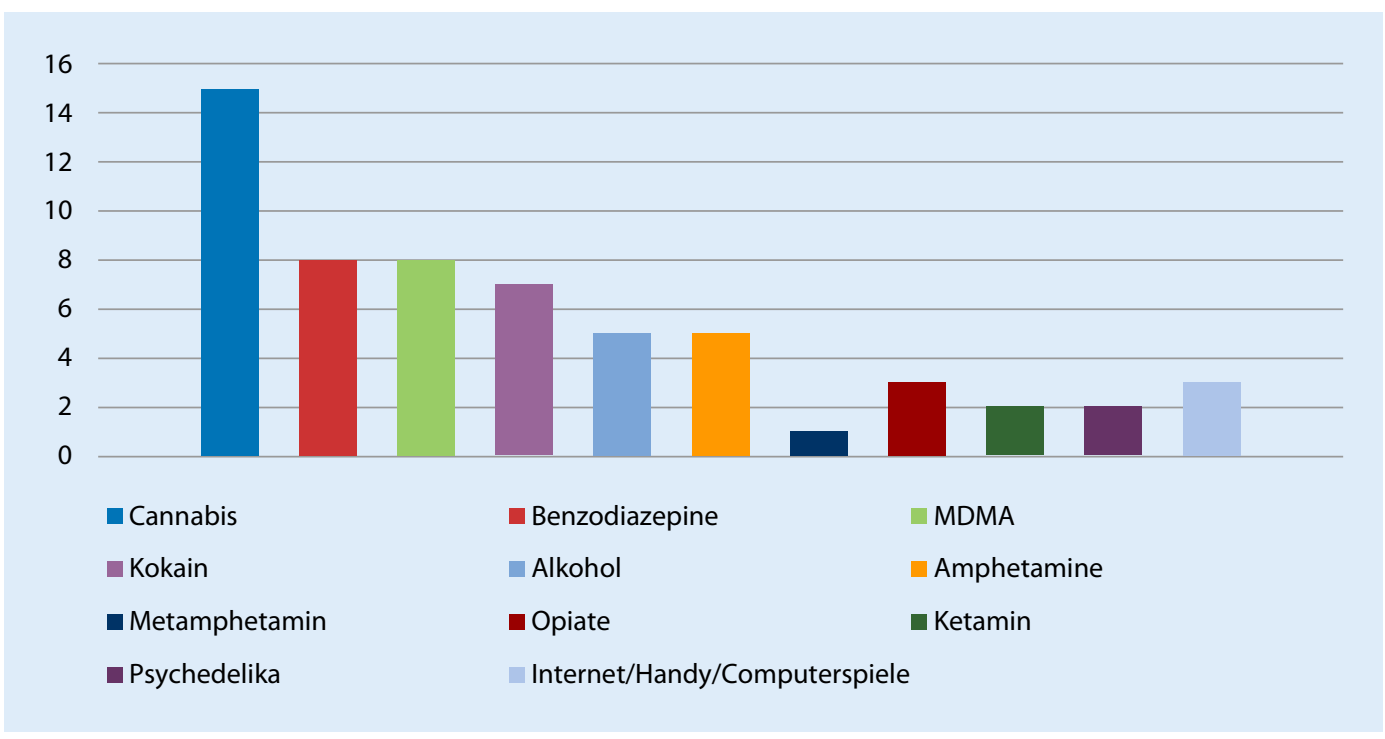

Abb. $2 \triangleleft$ Darstellung der von den Jugendlichen genannten konsumierten Substanzen, Mehrfachnennungen möglich. MDMA 3,4-Methylendioxy- $\mathrm{N}$-methylamphetamin

Hinsichtlich des Mediengebrauchs in der Freizeit stellt das Smartphone in aktuellen Befragungen das mit Abstand am meisten genutzte Angebot dar, wogegen Offline-Medien wie Zeitschriften, DVD oder Kinofilme bei Jugendlichen massiv an Stellenwert verloren haben. Während 2010 lediglich $9 \%$ der befragten Jugendlichen mobiles Internet als unverzichtbar ansahen, stimmte 2015 bereits fast die Hälfte ( $48 \%$ ) der befragten Jugendlichen dieser Aussage zu [14]. Dieser Siegeszug stimuliert einschlägige Forschung regelmäßig zu der Fragestellung, ob der Gebrauch neuer Technologien auf pathologische Art und Weise erfolgen könnte und ob ein solcher pathologischer Mediengebrauch schädlich für die psychi- sche Gesundheit von jungen Menschen sein könnte.

In den letzten Jahren hat sich eine breite Definition von Internetsucht durchgesetzt, die das Phänomen als „zunehmend exzessiver werdende und über einen längeren Zeitraum bestehende Beschäftigung mit spezifischen Internetinhalten, die im Laufe der Zeit andere Interessenfelder verdrängt, vom Betroffenen kaum noch bewusst kontrolliert werden kann und negative Konsequenzen in verschiedenen Bereichen nach sich zieht" beschreibt [15]. Bestimmte Internetinhalte wie Online-Computerspiele, soziale Netzwerke, Online-Glücksspiele oder Online-Pornographie scheinen dabei am häufigsten zu problematischer Nutzung zu führen.

Großangelegte Erhebungen auf europäischer Ebene erbrachten bisher relativ konstant den Befund, dass etwa 6-7\% der Jugendlichen im Querschnitt Hinweise auf einen potenziell schädlichen Gebrauch und 3-4\% Hinweise auf suchtartigen Gebrauch zeigen. Kriterien für eine Störung durch Spielen von Internet-Spielen (2013 im DSM-5 formuliert) wurden bei etwa $1,5 \%$ der europäischen Jugendlichen nachgewiesen [14].

Hinsichtlich der zugrundeliegenden Pathophysiologie weist suchtartiger Mediengebrauch in Bildgebungsstudien viele Parallelen zu Störungen durch Substanzgebrauch auf. Ähnlich wie bei 
stoffgebundenen Abhängigkeiten kann in Untersuchungen eine Beteiligung dopaminerger Belohnungssysteme sowie eine Beteiligung von Hirnarealen gezeigt werden, die für klassisches Suchtverhalten und Craving verantwortlich sind [14].

Neben dem menschlichen Urbedürfnis nach Kommunikation und sozialem Austausch sind vor allem technische Faktoren der Spieleprogrammierung wie die Möglichkeit eines Rollenwechsels, Experimentieren mit einer alternativen Identität (Avatar), regelmäßige Belohnungen, Gruppenzugehörigkeit, Verpflichtung (Gruppendruck), Wettbewerb und ein offenes Ende für die suchtartige Wirkung bestimmter Anwendungen verantwortlich. Es ist offensichtlich, dass diese Faktoren seitens der Industrie im Game-Design zur Kundenbindung von Jugendlichen bewusst instrumentalisiert werden [14].

In einer eigenen Studie an Tiroler Jugendlichen zeigte unsere Arbeitsgruppe, dass speziell junge Menschen mit psychischen Erkrankungen Schwierigkeiten haben, das Internet auf kompetente und nicht schädliche Art zu nutzen. Die Wahrscheinlichkeit für suchtartigen Internetgebrauch war bei Jugendlichen in stationärer psychiatrischer Behandlung im Vergleich zu einer Vergleichsstichprobe von Tiroler Schülern um das 7,8Fache erhöht. Darüber hinaus zeigen die Studienergebnisse, dass unter der Gruppe der internetabhängigen Jugendlichen bestimmte Probleme wie Mobbing, Identitätsschwierigkeiten oder Suizidalität signifikant häufiger vorkamen [16].

Im Gegensatz zu stofflichen Drogen ist im Fall des Internets eine dauerhafte Abstinenz nicht möglich und auch nicht sinnvoll, da junge Menschen in Ausbildung und Beruf aber auch privat auf diese Technologie angewiesen sind. Somit gilt es primär, einen vernünftigen und kompetenten Gebrauch zu erlernen und zu vermitteln. Dabei spielt die Mediensozialisation von Kindern eine große Rolle: Elterliche Laisser-faire-Haltung sowie die frühe Erfahrung von Trost, Schutz und positiver Emotionalität beim Benutzen von digitalen Medien scheinen negative prädiktive Faktoren für eine spätere dysfunktionale Nutzung darzustellen. Das gemeinsame Erarbeiten von Nutzungsregeln und gemeinsam gelebte OfflineZeiten sind andererseits wichtige Grundlagen im familiären Rahmen für die Entwicklung von Medienkompetenz [14].

Aus Längsschnittdaten wissen wir, dass suchtartiger Mediengebrauch nur selten ein langandauerndes Phänomen ist, und es ist oft im Querschnitt nicht einfach, zwischen einem intensiv ausgelebten Hobby eines Jugendlichen (Gamen), dessen Faszination nach einer gewissen Zeit wieder nachlässt, und einer tatsächlichen Sucht zu unterscheiden. Hilfreich ist hierbei sicherlich ein Heranziehen der oben genannten Definition. Bei vermeintlich oder tatsächlich problematischen Fällen ist deshalb neben einer genauen Erfassung des Konsummusters - eine Analyse der aktuellen Lebensumstände sowie eine psychiatrische Querschnittsdiagnostik anzuraten, um die Funktionalität der (pathologischen?) Mediennutzung auf Basis der derzeitigen Lebenssituation einschätzen zu können. Interventionen reichen je nach Ausprägungsgrad von ressourcen- und lösungsorientierter $\mathrm{Be}$ ratung bei leichten Fällen bis hin $\mathrm{zu}$ einer stationären Therapie mit initialer Medienabstinenz bei schweren Fällen [14].

\section{Suchtstation für Jugendliche: erste Erfahrungen}

Im Zuge der konzeptionellen Entwicklung der Abteilung für Kinder- und Jugendpsychiatrie, Psychotherapie und Psychosomatik am Standort Hall in Tirol wurde eine Station für Jugendliche mit substanzgebundenen und nicht substanzgebundenen Suchterscheinungen mit 6 Behandlungsplätzen geschaffen und Ende Februar 2018 eröffnet. Die Station ist als offener Behandlungsbereich konzipiert, eine freiwillige Bereitschaft zu Abstinenz ist Voraussetzung.

Die Analyse der ersten 27 Patienten (56\% weiblich, $44 \%$ mänlich; Durchschnittsalter 15,6 Jahre) zeigte interessante Trends: Zum einen überraschte uns das doch junge Einstiegsalter der Patienten, zum anderen der hohe Anteil an polytoxikoman konsumierenden Jugendlichen (• Abb. 1). Im Rahmen der
Hier steht eine Anzeige. Springer 
Suchtmittelanamnese wurden die Patienten aufgefordert, Drogen zu nennen, mit denen sie bereits Erfahrungen gemacht hatten. Hierbei fällt das extrem breite Substanzspektrum auf, wobei Cannabis, Benzodiazepine und MDMA die am häufigsten genannten Substanzen waren (• Abb. 2). Nahezu alle stationär aufgenommenen Patienten konsumierten Cannabis, nur mehr ein vergleichsweise geringer Anteil hatte Erfahrungen mit Opiaten. Auch der Prozentsatz der sich wegen einer reinen Alkoholproblematik vorstellenden Jugendlichen war vergleichsweise gering. Sorgen bereitet vor allem der Mischkonsum unter Benzodiazepin- oder Opiatbeteiligung: unter diesem Muster kam es in Tirol im laufenden Jahr zu mehreren Todesfällen bei jugendlichen Konsumentinnen.

Therapeutische Herausforderungen für das gesamte Team waren hohe Abbruchraten, Druck aus dem extramuralen Helfersystem (Wunsch nach Lösung) sowie fallweise stark ausgeprägte dissoziale Begleitphänomene (dealen, Sachbeschädigung, versteckter Konsum unter laufender Therapie), die sich bei ungünstiger Gruppenkonstellation verstärkten.

\section{Fazit für die Praxis}

Österreichische Jugendliche liegen beim Konsum der legalen Drogen AIkohol und Nikotin im europäischen Spitzenfeld. Ein weiterer Ausbau primärpräventiver Maßnahmen sowie ein konsequentes Rauchergesetz scheinen daher dringend geboten. Mit der Droge Internet ist ein neuartiges Gesundheitsproblem (Verhaltenssucht) bei Jugendlichen hinzugekommen, das unaufgeregter aber fundierter Diagnostik und unter Umständen therapeutischer Interventionen bedarf.

Im Fall von illegalen Drogen scheinen zusammenfassend folgende Punkte wichtig:

- Verbreiterung des Substanzspektrums beim jugendlichen Probierund Experimentierkonsum

- Europaweit Todesfälle durch hochdosiertes MDMA in Ecstasy-Tabletten
- Starker Rückgang des risikoreichen Opioidkonsums bei Jugendlichen in Österreich

- Cannabis im Jugendalter: komplexe Situation, Legalisierung kann für diese Altersgruppe nicht empfohlen werden, kaum Daten zu kontrollierter medizinischer Nutzung

- Problem hochriskanter Mischkonsum unter Benzodiazepin- und Opiatbeteiligung bei noch nicht manifester Abhängigkeit

- Todesfälle infolge Atemdepression (akzidentielle Überdosierung? Suizidversuch?)

- Abstinenz vs. Substitution

\section{Korrespondenzadresse}

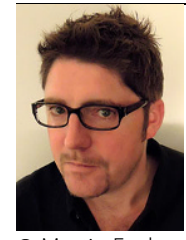

Dr. med. univ. Martin Fuchs Abteilung für Kinderund Jugendpsychiatrie, Psychotherapie und Psychosomatik, Tirol Kliniken Milser Straße 10,6060 Hall i. T. Österreich martin.fuchs@tirol-kliniken.at

Funding. Open access funding provided by University of Innsbruck and Medical University of Innsbruck.

\section{Einhaltung ethischer Richtlinien}

Interessenkonflikt. M. Fuchs gibt an, dass kein Interessenkonflikt besteht.

Dieser Beitrag beinhaltet keine von den Autoren durchgeführten Studien an Menschen oder Tieren.

Open Access. Dieser Artikel wird unter der Creative Commons Namensnennung 4.0 International Lizenz (http://creativecommons.org/licenses/by/4.0/deed de) veröffentlicht, welche die Nutzung, Vervielfältigung, Bearbeitung, Verbreitung und Wiedergabe in jeglichem Medium und Format erlaubt, sofern Sie den/die ursprünglichen Autor(en) und die Quelle ordnungsgemäß nennen, einen Link zur Creative Commons Lizenz beifügen und angeben, ob Änderungen vorgenommen wurden.

Hinweis des Verlags. Der Verlag bleibt in Hinblick auf geografische Zuordnungen und Gebietsbezeichnungen in veröffentlichten Karten und Institutsadressen neutral.

\section{Literatur}

1. Kemmesies U (2004) Zwischen Rausch und Realität: Drogenkonsum im bürgerlichen Milieu. Springer, Berlin Heidelberg

2. Strizek J, Anzenberger J, Kadlik A, Schmutterer I, Uhl A (2016) ESPAD Österreich. European School
Survey Project on Alcohol and other Drugs. Band 1: Forschungsbericht. Gesundheit Österreich, Wien

3. Horvath I, Anzenberger J, Busch M, GrabenhoferEggerth A, Schmutterer I, Strizek J, Tanios A, Weigl M (2018) Bericht zur Drogensituation 2018. Gesundheit Österreich, Wien.

4. Jochum M, Oeding J, Lackner D, Lienhart H (2017) Ecstasy-Intoxikation mit disseminierter intravasaler Gerinnung und Multiorganversagen. Anasthesiol Intensivmed Notfallmed Schmerzther 52(2):145-151

5. Hall W (2018) The future of the international drug control system and national drug prohibitions. Addiction 113(7):1210-1223

6. Freeman TP, Groshkova T, Cunningham A, Sedefov R, Griffiths P, Lynskey MT (2018) Increasing potency and price of cannabis in Europe, 2006-16. Addiction 2018 Dec 29. https://doi.org/10.1111/ add. 14525

7. Lubman DI, Cheetham A, Yücel M (2015) Cannabis and adolescent brain development. Pharmacol Ther 148:1-16

8. Levine A, Clemenza K, Rynn M, Lieberman J (2017) Evidence for the risks and consequences of adolescent cannabis exposure. J Am Acad Child Adolesc Psychiatry 56(3):214-225

9. Englund A, Freeman TP, Murray RM, McGuire P (2017) Can we make cannabis safer? Lancet Psychiatry 4(8):643-648

10. Filloux FM (2015) Cannabinoids for pediatric epilepsy? Up in smoke or real science? Transl Pediatr 4(4):271-282

11. Campbell CT, Phillips MS, Manasco K (2017) Cannabinoids in pediatrics. J Pediatr Pharmacol Ther 22(3):176-185

12. Bundeskriminalamt Österreich (2017) Lagebericht Suchtmittelkriminalität 2017. http:// bundeskriminalamt.at/302/files/Suchtmittel_17 Web.pdf.Zugegriffen:08.02.2019

13. Baumeister D, Tojo LM, Tracy DK (2015) Legal highs: staying on top of the flood of novel psychoactive substances. Ther Adv Psychopharmacol 5(2):97-132. https://doi.org/10.1177/ 2045125314559539

14. Fuchs M, Riedl D (2018) Dysfunktionaler und suchtartiger Internetgebrauch. In: Kothgasser OD, Felnhofer A (Hrsg) Klinische Cyberpsychologie und Cybertherapie. Utb facultas, Wien

15. Müller KW (2017) Internetsucht: Wie man sie erkennt und was man dagegen tun kann. Springer, Wiesbaden

16. Fuchs M, Riedl D, Bock A, Rumpold G, Sevecke K (2018) Pathological Internet use-an important comorbidity in child and adolescent psychiatry: prevalence and correlation patterns in a naturalistic sample of adolescent inpatients. Biomed Res Int. https://doi.org/10.1155/2018/1629147 